\title{
Análise das alterações posturais da coluna vertebral, dores musculoesqueléticas e função laboral em carteiros pedestres
}

\author{
Analysis of postural changes of the spine, musculoskeletal pain, and work activity \\ in walking letter carriers
}

\author{
Francini Porcher Andrade ${ }^{1} \bowtie$, Joel Hirtz do Nascimento Navarro ${ }^{1}$, Luisa Braga Jorge ${ }^{1}$, Cristiane Fernanda Gessinger
}

${ }^{1}$ Centro Universitário Metodista IPA. Porto Alegre, RS.

\section{RESUMO}

Objetivos: Avaliar as alterações posturais da coluna vertebral de carteiros pedestres com dores musculoesqueléticas e verificar sua associação com posturas adotadas durante a atividade laboral.

Métodos: $\mathrm{O}$ delineamento adotado foi um estudo transversal e descritivo realizado com carteiros pedestres de ambos os sexos, com dor musculoesquelética na coluna vertebral. Todos os indivíduos responderam a um questionário para apontar o local e intensidade da dor, realizaram uma avaliação postural fotogramétrica e foram submetidos a uma análise da função laboral. Para as análises em estratificação foi realizado o teste do qui-quadrado e para comparação dos ângulos da coluna vertebral nos indivíduos com e sem dor na mesma região foi realizado o teste $\mathrm{t}$ de Student. O nível de significância adotado foi $\mathrm{p} \leq 0,05$.

Resultados: Foram avaliados 20 carteiros pedestres. A média angular da curvatura lordótica dos sete indivíduos com dor na coluna cervical foi de $41,3 \pm 15,0^{\circ}$ e dos 13 indivíduos sem dor nessa região foi de $22,8 \pm 10,8^{\circ}(\mathrm{p}=0,005)$. Queixas de dor torácica ou lombar não tiveram associação significativa com o ângulo da curvatura cifótica e lordótica, respectivamente. Ao avaliar a postura do tronco dos carteiros no plano frontal observou-se que 16 indivíduos $(80 \%)$ apresentavam alguma forma de escoliose. Ao analisar a atividade laboral externa dos carteiros pedestres observou-se que oito indivíduos (40\%) carregavam a alça da bolsa de forma transpassada à esquerda, cinco (25\%) usavam a alça transpassada à direita, quatro $(20 \%)$ sobre o ombro direito e três $(15 \%)$ sobre o ombro esquerdo. Não houve diferença estatisticamente significativa entre as alterações posturais no plano frontal conforme a forma com que os carteiros carregavam a bolsa.

Conclusões: $\mathrm{Na}$ amostra de carteiros pedestres avaliada, houve associação da presença de dor na coluna cervical com o aumento da curvatura nessa região. Não foi encontrada associação significativa da alteração postural no plano frontal com a forma de carregar a bolsa durante a atividade laboral.

DESCRITORES: fotogrametria; dor musculoesquelética; postura; coluna vertebral; fisioterapia.

\section{ABSTRACT}

Aims: To assess postural changes of the spine in walking letter carriers with musculoskeletal pain and check their association with positions taken during work activity.

Methods: This was a cross-sectional descriptive study of both male and female letter carriers with musculoskeletal pain in the spine. All subjects completed a questionnaire to indicate the location and severity of pain and were subjected to a photogrammetric analysis of their posture and of their work activity. The chi-square test was applied for stratified analysis and Student's t test was used to compare spinal angles in individuals with and without pain in the same region. A p value equal to or less than 0.05 was considered to be statistically significant.

Results: A total of 20 walking letter carriers were assessed. The mean lumbar lordotic angle of the seven patients with pain in the cervical spine was $41.3 \pm 15.0^{\circ}$ while in the 13 individuals without pain in this region it was $22.8 \pm 10.8^{\circ}(\mathrm{p}=0.005)$. The presence of thoracic or lumbar pain had no significant association with the angle of the kyphotic and lordotic curvature, respectively. The assessment of the frontal plane posture of letter carriers revealed that $16(80 \%)$ had some kind of scoliosis. The analysis of work-relatedness indicated that eight (40\%) strapped the mail bag crosswise to the left, five $(25 \%)$ wore it crosswise to the right, four $(20 \%)$ slung the mail bag over their right shoulder, and three $(15 \%)$ carried it on their left shoulder. No statistically significant difference was observed in postural changes on the frontal plane associated with how the mail bags were carried.

Conclusions: There was a significant association between the presence of pain in the cervical spine and a more pronounced curvature in this region.

KEY WORDS: photogrammetry; musculoskeletal pain; posture; spine; physical therapy specialty. 
Abreviaturas: EAV, escala análogo visual; OWAS, Ovako Working Posture Analysing System; RULA, Rapid Upper Limb Assessment.

\section{INTRODUÇÃO}

Os segmentos ósseos e musculares são elementos que garantem a organização e a coordenação dos movimentos corporais a fim de proporcionar uma boa postura. As forças que movem e estabilizam o corpo também oferecem potencial para deformá-lo, e o uso incorreto da postura resulta em sobrecarga das estruturas que promovem o suporte corporal, podendo causar lesões musculoesqueléticas. A todo o momento, diferentes posturas são adotadas inconscientemente pelo corpo, a fim de obter conforto na execução de determinadas atividades [1-3]. As atividades que exigem esforço corporal para transportar cargas externas podem ocasionar lesões por sobrecarga corporal se o estresse físico exceder o limite individual da pessoa, dando origem às alterações posturais e/ou dores musculoesqueléticas $[4,5]$.

Os carteiros pedestres realizam diferentes atividades laborais ao longo da jornada de trabalho, executando a maioria delas na postura vertical, e desta forma, exigindo certo esforço da coluna vertebral. Geralmente, metade da jornada do trabalho desses profissionais é interna, quando trabalham em postura vertical estática separando cartas, enquanto a outra metade é preenchida com caminhadas ao ar livre, quando realizam o transporte manual de cargas contidas em uma bolsa, cuja alça foi confeccionada para ser utilizada de forma transpassada pelo tronco do individuo [3-5]. Uso excessivo de força, repetição de movimentos e posturas inadequadas durante as atividades laborais são rotinas básicas dos carteiros pedestres e são consideradas as principais causas de lesões musculoesqueléticas nesses trabalhadores, principalmente da coluna vertebral, influenciando a taxa de absenteísmo nas empresas e a redução da vida útil do trabalhador [6-9].

Um fisioterapeuta atuante na saúde do trabalhador pode analisar os comportamentos posturais adotados por diferentes profissionais, relacionando-as com possíveis alterações posturais na coluna vertebral. A avaliação postural e a análise das posturas utilizadas no trabalho permitem identificar comprometimentos musculoesqueléticos, e assim, prevenir e minimizar desequilíbrios musculares [10].

Existem alguns métodos utilizados para analisar a atividade laboral de determinadas classes de trabalhadores, tais como o método OWAS (Ovako Working Posture Analysing System), elaborado para trabalhadores de indústrias de aço, e o método RULA (Rapid Upper Limb Assessment), focado na avaliação dos membros superiores [11]. Contudo, estudos envolvendo carteiros pedestres são escassos, o que justifica a falta de instrumentos adequados para a análise da atividade laboral desses trabalhadores. Um dos métodos para realizar a avaliação postural de forma rápida e facilitada é a fotogrametria computadorizada, caracterizada por ser um recurso não invasivo e que emprega o uso da fotointerpretação por meio de programas de computador $[1,2]$.

Desta forma, o objetivo deste estudo foi avaliar as alterações posturais da coluna vertebral de carteiros pedestres com dores musculoesqueléticas e verificar sua associação com posturas adotadas durante a atividade laboral externa.

\section{MÉTODOS}

Realizou-se um estudo transversal que incluiu carteiros pedestres que atuavam no Centro de Distribuição Domiciliar-Centro da Empresa Brasileira de Correios e Telégrafos de Porto Alegre, Rio Grande do Sul. A coleta dos dados ocorreu no período de agosto a dezembro de 2012. A pesquisa foi aprovada pelo Comitê de Ética em Pesquisa do Centro Universitário Metodista IPA sob o protocolo 106/2012. Todos os participantes assinaram um termo de consentimento livre e esclarecido.

A seleção da amostra foi por conveniência e foram incluídos apenas indivíduos com dores musculoesqueléticas na coluna vertebral e excluídos aqueles que haviam sofrido traumas graves há menos de dois anos ou que haviam sofrido procedimentos cirúrgicos na coluna vertebral. Os carteiros pedestres voluntários a participar do estudo foram inicialmente convocados para avaliação por meio de um questionário elaborado pelos autores da pesquisa para a caracterização da amostra. Esse questionário continha dados como: idade, sexo, peso, altura e índice de massa corporal [12], percepção do peso da bolsa carregada durante a jornada externa de trabalho, tempo na função de carteiro pedestre, prática de exercício físico, local da dor, intensidade da dor, características da dor quanto à permanência durante o dia, relato de traumas graves há menos de dois anos e história de procedimentos cirúrgicos na coluna vertebral.

A percepção do peso da bolsa foi avaliada por meio de uma escala análogo visual (EAV) [13], considerando zero a ausência de peso e 10 o peso máximo suportado 
pelo indivíduo. Para a marcação do local da dor na coluna vertebral utilizou-se uma figura do tronco do diagrama de Corlett na vista posterior [13], sendo que o indivíduo deveria marcar todas as regiões da coluna vertebral em que sentia dores. Para quantificar a intensidade da dor foi usada uma EAV, sendo zero a ausência de dor e 10 a maior dor possível [14].

Após o preenchimento do questionário, era realizada a pesagem da bolsa e o registro de campo do trabalho externo dos carteiros (atividades realizadas para a entrega das correspondências), para verificar as posturas adotadas durante essas atividades. Para isso, foi utilizado um roteiro elaborado pelos autores da pesquisa, contendo os seguintes itens: 1) forma em que carrega a alça da bolsa; 2) hábito de trocar de lado a alça da bolsa; 3) elevação do ombro que carrega a alça da bolsa; 4) hábito de soltar a bolsa no chão durante o trabalho; 5) hábito de apanhar a bolsa do chão realizando flexão anterior de tronco e semiflexão de joelhos. As posturas observadas foram documentadas por fotografias e vídeos realizados com uma câmera digital (Samsung ${ }^{\circledR}$ modelo PL120).

Após o registro de campo foi realizada uma avaliação postural por meio da fotogrametria, utilizando um espaço físico com fundo branco opaco, demarcações no piso com fita amarela em formato quadrangular contendo $30 \mathrm{~cm}^{2}$ e posicionado a $15 \mathrm{~cm}$ da parede para identificar o local onde o indivíduo deveria situar-se descalço. Os pontos anatômicos foram identificados com marcadores de superfície sempre pelo mesmo avaliador, utilizando etiquetas autoadesivas de cor azul $\left(\right.$ Pimaco $\left.^{\circledR}\right)$ com $12 \mathrm{~mm}$ de diâmetro. Sobre cada etiqueta foi fixada uma esfera de isopor em cor azul presa com fita dupla face nas seguintes áreas localizadas por meio de palpação: processos espinhosos de C4, C7, T7, T12, L3 e L5, espinhas ilíacas ântero-superiores, espinhas ilíacas póstero-superiores e póstero-inferiores, articulações esternoclaviculares, articulações acromioclaviculares e ângulo inferior das escápulas $[1,2]$. Sobre cada esfera posicionada nos processos espinhosos da coluna vertebral foi colocado um palito de madeira a fim de identificar a posição dos marcadores no plano sagital. Para a calibração da imagem foi utilizado um fio de prumo com duas demarcações separadas por $10 \mathrm{~cm}$.

Para a captação da imagem nos planos frontal e sagital, era solicitado que as mulheres utilizassem roupas de banho na parte superior do corpo, sendo que todos os indivíduos deveriam abaixar a calça do uniforme de trabalho a uma altura que fosse possível a visualização da espinha ilíaca póstero inferior.
Era solicitado que os indivíduos permanecessem na posição mais natural possível. O registro fotográfico da postura foi feito com a câmera digital sobre um tripé (Manfrotto $^{\circledR}$, modelo 190XPROB) posicionado a $1 \mathrm{~m}$ de altura do chão e a 2,40 $\mathrm{m}$ de distância do participante [1]. Para a análise das posturas foi utilizado o software para avaliação postural SAPO ${ }^{\circledR}$, versão 0.68 .

Para determinação de escoliose foram considerados o desalinhamento da coluna vertebral de acordo com os pontos demarcados, o desnivelamento de ombros anteriormente, o desnivelamento de escápulas posteriormente e o desnivelamento de pelve anteriormente e posteriormente. As escolioses foram classificadas com dupla curvatura na presença da combinação de todas as variáveis acima [15]. Foi considerada uma margem de erro de $0,5 \mathrm{~cm}$ de diferença nas elevações da pelve e escápula.

Quanto ao alinhamento frontal na vista posterior da coluna vertebral, determinou-se que quando houvesse equilíbrio de uma das cinturas, pélvica ou escapular, era utilizado uma margem de erro de $0,5 \mathrm{~cm}$, considerando a distância na linha horizontal de dois pontos demarcados consecutivamente, ou seja, se a distância horizontal de $\mathrm{C} 4$ diferisse até $0,5 \mathrm{~cm}$ de $\mathrm{C} 7$, era considerado alinhamento vertebral.

As variáveis contínuas foram descritas por média e desvio padrão e as variáveis ordinais por frequência absoluta e relativa. Para verificar a normalidade dos dados, foi realizado o teste de Shapiro-Wilk. Para realizar as análises em estratificação foi realizado o teste de Qui-Quadrado. Para comparação dos ângulos da coluna vertebral nos indivíduos com e sem dor na mesma região foi realizado o teste $\mathrm{t}$ de Student. Para correlação do peso da bolsa com os ângulos da coluna vertebral, bem como da intensidade de dor com a percepção do peso da bolsa foi realizado o teste de Correlação de Pearson. Os dados foram armazenados e analisados no software IBM SPSS Statistics V 20.0, adotando o nível de significância $p \leq 0,05$.

\section{RESULTADOS}

O Centro de Distribuição Domiciliar-Centro contava no período do estudo com 33 carteiros pedestres de ambos os sexos, com média de idade 41,5 $\pm 10,4$ anos. Destes, $23(69,6 \%)$ apresentavam dor na coluna vertebral. Três foram excluídos do estudo por terem realizado procedimento cirúrgico na coluna vertebral (artrodese). Portanto, 20 carteiros pedestres de ambos os sexos se encaixaram nos critérios do estudo e todos esses aceitaram participar. A caracterização da amostra está representada na Tabela 1. 
Tabela 1. Características da amostra constituída por carteiros pedestres que apresentavam algum tipo de dor musculoesquelética na coluna vertebral. Porto Alegre, RS, 2012.

\begin{tabular}{|c|c|}
\hline Variáveis & $\mathbf{n}=\mathbf{2 0}$ \\
\hline Sexo masculino* & $11(64,7 \%)$ \\
\hline Idade (anos) $\dagger$ & $41,5 \pm 10,4$ \\
\hline Peso $(\mathrm{kg}) \dagger$ & $75,1 \pm 12,0$ \\
\hline $\mathrm{IMC}^{+}$ & $26,6 \pm 4,2$ \\
\hline Peso da bolsa $(\mathrm{kg}) \dagger$ & $6,7 \pm 1,8$ \\
\hline Percepção do peso da bolsa (EAV $0-10)+$ & $7,6 \pm 1,4$ \\
\hline Percepção de dor (EAV 0-10)† & $6,1 \pm 2,4$ \\
\hline Tempo na função (anos) $†$ & $12,6 \pm 7,9$ \\
\hline Pratica atividade física* & $1(5 \%)$ \\
\hline \multicolumn{2}{|l|}{ Local de dor } \\
\hline Coluna cervical* & $7(35 \%)$ \\
\hline Coluna torácica* & $8(40 \%)$ \\
\hline Coluna lombar* & $14(70 \%)$ \\
\hline \multicolumn{2}{|l|}{ Presença de dor } \\
\hline Persistente o dia inteiro* & $13(65 \%)$ \\
\hline Somente no trabalho interno* & $3(15 \%)$ \\
\hline Após o trabalho* & $3(15 \%)$ \\
\hline Somente no trabalho externo* & $1(5 \%)$ \\
\hline
\end{tabular}

* Número (porcentagem).

† Média \pm desvio padrão.

IMC, índice de massa corporal; EAV, escala análogo visual.

Ao avaliar a postura do tronco dos carteiros no plano frontal observou-se que quatro indivíduos (20\%) apresentaram escoliose sinistroconvexa, quatro $(20 \%)$ escoliose dextroconvexa, quatro $(20 \%)$ escoliose dextroconvexa dorsal e sinistroconvexa lombar, quatro (20\%) escoliose sinistroconvexa dorsal e dextroconvexa lombar e quatro indivíduos $(20 \%)$ apresentaram a postura neutra no plano frontal. Ao analisar a atividade laboral externa dos carteiros pedestres observou-se que oito indivíduos (40\%) carregavam a alça da bolsa de forma transpassada à esquerda, cinco $(25 \%)$ usavam a alça transpassada à direita, quatro $(20 \%)$ sobre o ombro direito e três $(15 \%)$ sobre o ombro esquerdo. Não houve diferença estatisticamente significativa ao comparar as alterações posturais no plano frontal entre os cinco subgrupos (quatro subgrupos com algum tipo de escoliose e um sem escoliose) e a forma com que os carteiros carregavam a bolsa.

Quando comparada a presença de dor na coluna cervical com a média angular no plano sagital da respectiva região, foi encontrado resultado estatisticamente significativo, sugerindo que os graus mais elevados da curvatura cervical estão relacionados com a presença de dor nesse local. Já nas dores localizadas nas regiões torácica e lombar não foi encontrada relação com as curvaturas cifóticas ou lordóticas nas respectivas regiões (Tabela 2).

Tabela 2. Relação entre os ângulos sagitais das curvas nos diversos segmentos da coluna vertebral e a presença da dor no mesmo local, em carteiros pedestres que apresentavam algum tipo de dor musculoesquelética na coluna vertebral. Porto Alegre, RS, 2012.

\begin{tabular}{|c|c|c|}
\hline Segmento da coluna & Ângulos * & pt \\
\hline \multicolumn{3}{|l|}{ Cervical $(n=20)$} \\
\hline Com dor na coluna cervical $(n=7)$ & $41,3 \pm 15,0^{\circ}$ & 0,005 \\
\hline Sem dor na coluna cervical $(n=13)$ & $22,8 \pm 10,8^{\circ}$ & \\
\hline \multicolumn{3}{|l|}{ Torácica $(n=20)$} \\
\hline Com dor na coluna torácica $(n=8)$ & $49,5 \pm 12,99$ & 0,860 \\
\hline Sem dor na coluna torácica $(n=12)$ & $48,5 \pm 14,7^{\circ}$ & \\
\hline \multicolumn{3}{|l|}{ Lombar $(n=20)$} \\
\hline Com dor na coluna lombar $(n=4)$ & $26,3 \pm 12,1^{\circ}$ & 0,670 \\
\hline Sem dor na coluna lombar $(n=6)$ & $29,1 \pm 16,1^{\circ}$ & \\
\hline
\end{tabular}

* Em graus (média \pm desvio padrão).

† Teste t de Student.

Ao avaliar a correlação entre a média do peso da bolsa $(6,7 \pm 1,8 \mathrm{~kg})$ dos carteiros pedestres com a média dos ângulos no plano sagital da coluna cervical $(\mathrm{r}=-0,42 ; \mathrm{p}=0,06)$, torácica $(\mathrm{r}=-0,28 ; \mathrm{p}=0,21)$ e lombar $(\mathrm{r}=0,06 ; \mathrm{p}=0,79)$ houve ausência de correlação, ou seja, o peso da bolsa não se correlacionou com a intensidade das curvaturas da coluna no plano sagital. Também não foi encontrada correlação entre a intensidade de dor e a percepção do peso da bolsa $(r=0,20 ; p=0,38)$.

\section{DISCUSSÃO}

Analisando as características da população estudada, foi possível verificar que a maioria (quase $70 \%$ ) dos carteiros pedestres apresentava dor na região da coluna lombar. A dor na lombar leva à incapacidade funcional pelo impacto que exerce na rotina de um trabalhador e seu aparecimento está relacionado tanto ao estilo de vida como aos hábitos laborais, no entanto, a relação de causalidade entre esses fatores de risco e o desenvolvimento de dor lombar permanece discutível $[16,17]$.

A região lombar é a principal área de suporte corporal, e qualquer carga externa ao corpo pode gerar sobrecarga nessa região [18]. O estudo de Jensen et al. [16], que avaliou o impacto dos fatores de riscos laborais no desenvolvimento da lombalgia em 2.235 profissionais da área da saúde, apontou que os trabalhadores com elevada carga de trabalho físico apresentavam maior risco de desenvolver lombalgia [16]. Carteiros pedestres apresentam elevada carga 
de trabalho, uma vez que realizam suas atividades internas durante um turno, com movimentos repetitivos na postura ortostática estática, comprometendo a irrigação muscular e causando fadiga [19], enquanto no outro turno, de atividades externas, realizam o transporte manual de cargas.

No presente estudo, quando estratificada a amostra pelos diferentes tipos de escoliose, não houve relação com a forma de carregar a alça da bolsa pelos carteiros pedestres e uma possível explicação para este fato foi a estratificação em muitas categorias, resultando em um número pequeno de sujeitos por grupo. Diferentemente desta pesquisa, o estudo de Negrini e Negrini [20], que avaliou a postura de estudantes enquanto carregavam peso, concluiu que o transporte de cargas solicitava variações da postura, sendo que na existência de um peso externo assimétrico junto ao corpo, há elevação do ombro, juntamente com um desvio lateral da coluna vertebral, o que favorece o aparecimento das escolioses. Araújo et al. [21] também comentam que o carregamento de peso assimétrico favorece os desvios laterais devido à tensão dos músculos contralaterais do tronco, na tentativa de manter o alinhamento.

Foi possível constatar que os carteiros pedestres que referiam dor na coluna cervical foram aqueles que apresentaram maiores angulações dessa região no plano sagital, sugerindo uma tendência à hiperlordose. Não foram encontrados na literatura estudos utilizando a biofotogrametria que definam valores angulares adequados para estabelecer diagnósticos das curvaturas da coluna vertebral no plano sagital. Dessa forma, não é possível afirmar que esses indivíduos possuíam hiperlordose cervical [22-24]. Diferentemente do presente estudo, o de Yip et al. [25] avaliou 62 pacientes com cervicalgia e encontrou correlação desse sintoma com menores angulações da cervical no plano sagital. É possível inferir que a diferença entre os resultados seja devida ao fato de que naquela pesquisa os participantes não apresentavam elevada carga de trabalho com repetição de movimentos [25].
O estudo de Negrini e Negrini [20] concluiu que o carregamento de um peso extra, mesmo que seja simétrico, solicita variações posturais devido à fadiga muscular originada por esta ação. Quando se carrega um volume externo junto ao corpo, há flexão para frente, a fim de manter o centro de gravidade dentro de uma área de apoio. Por este motivo, pode haver um alongamento da coluna vertebral quando se carrega peso, o que pode gerar redução das curvaturas no plano sagital [20].

Este estudo evidenciou que o peso, assim como a sua percepção pelo indivíduo, da bolsa carregada pelos carteiros pedestres não implicou na intensidade da dor. Silva et al. [26], ao verificarem a prevalência de dor lombar em indivíduos que carregavam peso, também não encontraram relação entre essas variáveis, corroborando com este estudo. No estudo de Schneider et al. [27], o transporte de peso e o trabalho fisicamente extenuante estiveram associados a um risco significativamente maior de queixa de dor nas costas em trabalhadores de diferentes funções. O manejo de cargas durante o trabalho pode representar risco ao sistema musculoesquelético quando exceder os limites individuais, devido principalmente à sobrecarga na região da coluna vertebral [4]. As recomendações para o transporte manual de peso é que ele não ultrapasse $15 \%$ do peso corporal [28].

Como limitações deste estudo pode-se considerar o número amostral pequeno, bem como o fato de não terem sido avaliados os carteiros pedestres sem dores musculoesqueléticas na coluna vertebral, inviabilizando a comparação entre grupos conforme a presença ou não de dor. Sugere-se a realização de mais estudos envolvendo carteiros pedestres, que incluam amostras maiores e indivíduos com e sem dor.

Mesmo considerando essas limitações, foi possível concluir que é bastante prevalente a presença de dor musculoesquelética em carteiros pedestres. Além disso, a angulação lordótica da coluna cervical apresentou relação com a presença de dor na mesma região.

NOTA

Agradecimentos

À Empresa Brasileira de Correios e Telégrafos pela contribuição nesta pesquisa, autorizando os seus profissionais a fazerem parte do estudo, bem como contribuindo com o espaço físico para as avaliações realizadas.

\section{REFERÊNCIAS}

1. Iunes DH, Cecílio MBB, Dozza MA, Almeida PR. Análise quantitativa do tratamento da escoliose com o método klapp por meio da biofotogrametria computadorizada. Rev Bras Fisioter. 2010;14(2):133-40. http://dx.doi.org/10.1590/S1413-35552010005000009 
2. Figueiredo RV, Amaral AC, Shimano AC. Fotogrametria na identificação de assimetrias posturais em cadetes e pilotos da Academia da Força Aérea Brasileira. Rev Bras Fisioter. 2012;16(1):54-60. http://dx.doi.org/10.1590/S1413-35552012000100010

3. Balasubramanian V, Adalarasu K, Regulapati R. Comparing dynamic and stationary standing postures in an assembly task. Int J Ind Ergon. 2009;39(2):649-54. http://dx.doi.org/10.1016/j.ergon.2008.10.017

4. Carregaro RL, Coury HJCG. Análise biomecânica da coluna durante manuseio de cargas realizado por sujeitos experientes e inexperientes. Fisioter e Pesq. 2007;14(2):57-64.

5. Rodrigues S, Teodori RM. Distribuição da Força Plantar e Oscilação do centro de pressão em relação ao peso e posicionamento do material escolar. Rev Bras Fisioter. 2008;12(1):43-8. http://dx.doi.org/10.1590/s1413-35552008000100009

6. Magnago TSBS, Lisboa MTL, Souza IEO, Moreira MC. Distúrbios Musculo-Esqueléticos em Trabalhadores de Enfermagem: Associação com Condições de Trabalho. Rev Bras Enferm. 2007;60(6):701-05. http://dx.doi.org/10.1590/S0034-71672007000600015

7. Roffey DM, Wai EK, Bishop P, Kwon BKK, Dagenais S. Causal assessment of occupational standing or walking and low back pain: results of a systematic Review. Spine J. 2010;10(3):262-72. http://dx.doi.org/10.1016/j.spinee.2009.12.023

8. Cardoso JP, Ribeiro IQB, Araújo TM, Carvalho FM, Reis EJFB. Prevalência de dor musculoesquelética em professores. Rev Bras Epidemiol. 2009;12(4):604-14. http://dx.doi.org/10.1590/S1415-790X2009000400010

9. Coury HJCG, Moreira RFC, Dias NB. Efetividade do exercício físico em ambiente ocupacional para controle da dor cervical, lombar e do ombro: uma revisão sistemática. Rev Bras Fisioter. 2009;13(6):461-79. http://dx.doi.org/10.1590/S1413-35552009000600002

10. Neumann WP, Wells RP, Norman RW, Frank J, Shannon H, Kerr MS. A posture and load sampling approach to determining low-back pain risk in occupational settings. Int J Ind Ergon. 2001;27(2):65-77. http://dx.doi.org/10.1016/S0169-8141(00)00038-X

11. Xu YW, Cheng AS. An onsite ergonomics assessment for risk of work-related musculoskeletal disorders among cooks in a Chinese restaurant. Work. 2014;48(4):539-45. http://dx.doi.org/10.3233/WOR-131805

12. Nilsen TI, Holtermann A, Mork PJ. Physical exercise, body mass index, and risk of chronic pain in the low back and neck/shoulders: longitudinal data from the Nord-Trondelag Health Study. Am J Epidemiol. 2011;174(3):267-73. http://dx.doi.org/10.1093/aje/kwr087

13. de Freitas-Swerts FC, Robazzi ML. The effects of compensatory workplace exercises to reduce work-related stress and musculoskeletal pain. Rev Lat Am Enfermagem. 2014;22(4):629-36. http://dx.doi.org/10.1590/0104-1169.3222.2461

14. Kim HJ, Yu SH. Effects of complex manual therapy on PTSD, pain, function, and balance of male torture survivors with chronic low back pain. J Phys Ther Sci. 2015;27(9):2763-66. http://dx.doi.org/10.1589/jpts.27.2763

15. Döhnert MB, Tomasi E. Validade da fotogrametria computadorizada na detecção de escoliose idiopática adolescente. Rev Bras Fisioter. 2008;12(4):290-97. http://dx.doi.org/10.1590/S1413-35552008000400007

16. Jensen JN, Holtermann A, Clausen T, Mortensen OS, Carneiro IG, Andersen LL. The greatest risk for low-back pain among newly educated female health care workers; body weight or physical work load? BMC Musculoskelet Disord. 2012;13:87. http://dx.doi.org/10.1186/ 1471-2474-13-87

17. Murtezani A, Hundozi H, Orovcanec N, Berisha M, Meka V. Low back pain predict sickness absence among power plant workers. Indian J Occup Environ Med. 2010;14(4):49-53. http://dx.doi.org/10.4103/0019-5278.72241

18. Oliveira AGS, Nakke HA, Alencar JF. Riscos biomecânicos posturais em trabalhadores de uma serraria. Fisiot Pesq. 2009;16(1):28-33.

19. Berenguer FA, E Silva DAL, De Carvalho CC. Influência da posição ortostática na ocorrência de sintomas e sinais clínicos de venopatias de membros inferiores em trabalhadores de uma gráfica na cidade do Recife-PE. Rev Bras Saúde Ocup. 2011;36(123):153-61. http://dx.doi.org/10.1590/S0303-76572011000100016

20. Negrini S, Negrini A. Postural effects of symmetrical and asymmetrical loads on the spines of schoolchildren. Scoliosis. 2007;9:2-8. http://dx.doi.org/10.1186/1748-7161-2-8

21. Araújo MEA, Silva EB, Vieira SAC, Mello DB, Dantas EHM. Redução da dor crônica associada à escoliose não estrutural, em universitárias submetidas ao método Pilates. Motriz. 2010;16(4):958-66. http://dx.doi.org/10.5016/1980-6574.2010v16n4p958

22. Iunes DH, Salgado HS, Moura IC, Oliveira AS, Bevilaqua-grossi D. Confiabilidade intra e interexaminadores e repetibilidade da avaliação postural pela fotogrametria. Rev Bras Fisioter. 2005;9(3):e327.

23. Carnielli AL, Ninello DA, Fereira DMA, Pachioni CAS. Análise da cifose torácica e lordose lombar em indivíduos com escoliose idiopática. Rev Eletr Fisiot FCT/UNESP. 2009;1(1):22-45.

24. Souza JA, Pasinasto F, Basso D, Corrêa ECR, Silva AMT. Biofotogrametria confiabilidade das medidas do protocolo do software para avaliação postural (SAPO). Rev Bras Cineantropom Desempenho Hum. 2011;13(4):299-05.

25. Yip CHT, Chiu TTW, Poon ATK. The relationship between head posture and severity and disability of patients with neck pain. Manual Therapy. 2008;13(2):148-54. http://dx.doi.org/10.1016/j.math.2006.11.002

26. Silva MC, Fassa ACG, Valle NCJ. Dor lombar crônica em uma população adulta do Sul do Brasil: prevalência e fatores associados. Cad. Saúde Pública. 2004;20(2):377-85. http://dx.doi.org/10.1590/S0102-311X2004000200005

27. Schneider S, Lipinski S, Schiltenwolf M. Occupations associated with a high risk of self-reported back pain: representative outcomes of a back pain prevalence study in the Federal Republic of Germany. Eur Spine J. 2006;15(6):821-33. http://dx.doi.org/10.1007/s00586-0051015-2

28. Chow DHK, Kwok MLY, Au-yang ACK, Holmes AD, Cheng JCY, Yao FYD, Wong MS. The effect of backpack load on the gait of normal adolescent girls. Ergonomics. 2005;48(6):642-56. http://dx.doi.org/10.1080/00140130500070921 